\title{
Longitudinal Viscous Flow in Granular Gases
}

\author{
Andrés Santos \\ Departamento de Física, Universidad de Extremadura, E-06071 Badajoz, Spain
}

\begin{abstract}
The flow characterized by a linear longitudinal velocity field $u_{x}(x, t)=a(t) x$, where $a(t)=a_{0} /\left(1+a_{0} t\right)$, a uniform density $n(t) \propto a(t)$, and a uniform temperature $T(t)$ is analyzed for dilute granular gases by means of a BGK-like model kinetic equation in $d$ dimensions. For a given value of the coefficient of normal restitution $\alpha$, the relevant control parameter of the problem is the reduced deformation rate $a^{*}(t)=a(t) / v(t)$ (which plays the role of the Knudsen number), where $v(t) \propto n(t) \sqrt{T(t)}$ is an effective collision frequency. The relevant response parameter is a nonlinear viscosity function $\eta^{*}\left(a^{*}\right)$ defined from the difference between the normal stress $P_{x x}(t)$ and the hydrostatic pressure $p(t)=n(t) T(t)$. The main results of the paper are: (a) an exact first-order ordinary differential equation for $\eta^{*}\left(a^{*}\right)$ is derived from the kinetic model; (b) a recursion relation for the coefficients of the Chapman-Enskog expansion of $\eta^{*}\left(a^{*}\right)$ in powers of $a^{*}$ is obtained; (c) the Chapman-Enskog expansion is shown to diverge for elastic collisions $(\alpha=1)$ and converge for inelastic collisions $(\alpha<1)$, in the latter case with a radius of convergence that increases with inelasticity; (d) a simple approximate analytical solution for $\eta^{*}\left(a^{*}\right)$, hardly distinguishable from the numerical solution of the differential equation, is constructed.
\end{abstract}

Keywords: Chapman-Enskog expansion, Granular gases, Rheological properties PACS: $45.70 . \mathrm{Mg}, 05.20 . \mathrm{Dd}, 47.50 .-\mathrm{d}, 51.10 .+\mathrm{y}$

\section{INTRODUCTION}

A granular gas is a large collection of mesoscopic or macroscopic particles which collide inelastically and are maintained in a fluidized state. The prototypical model of a granular gas consists of a dilute system of smooth inelastic hard spheres characterized by a constant coefficient of normal restitution $\alpha<1$ [1]. A kinetic theory description based on the Boltzmann equation for inelastic collisions has proven its relevance for the understanding of the main properties of dilute granular gases, such as non-equipartition of energy and high-velocity tails in uniform states or non-Newtonian transport properties in non-uniform steady states.

The kinetic theory definition of nonequilibrium temperature $T$ as a measure of the mean kinetic energy (in the Lagrangian frame) per particle can be directly extended to granular gases (except that it is customary to measure $T$ in energy units, i.e., to take the Boltzmann constant equal to unity). The key difference with respect to normal gases is that the inelastic character of collisions gives rise to an energy sink, so that the temperature change per unit time due to collisions is $\left.\partial_{t} T\right|_{\text {coll }}=-\zeta T$, where $\zeta$ is the so-called cooling rate. It is given (in the Maxwellian approximation) by

$$
\zeta=\frac{d+2}{4 d}\left(1-\alpha^{2}\right) \omega, \quad \omega \equiv \frac{8 \pi^{(d-1) / 2}}{(d+2) \Gamma(d / 2)} n \sigma^{d-1} \sqrt{\frac{T}{m}},
$$

where $d$ is the dimensionality of the system, $n$ is the number density, $\sigma$ is the diameter of a sphere, and $m$ is its mass. Application of the Chapman-Enskog (CE) method to the inelastic Boltzmann equation allows one to derive the Navier-Stokes (NS) constitutive equations for inelastic hard spheres [2]. In particular, Newton's law reads

$$
P_{i j}=p \delta_{i j}-\eta_{\mathrm{NS}}\left(\frac{\partial u_{i}}{\partial x_{j}}+\frac{\partial u_{j}}{\partial x_{i}}-\frac{2}{d} \nabla \cdot \mathbf{u} \delta_{i j}\right)
$$

where $P_{i j}$ is the pressure tensor, $p=(1 / d) \operatorname{Tr} \mathrm{P}=n T$ is the hydrostatic pressure, $\mathbf{u}$ is the flow velocity, and $\eta_{\mathrm{NS}}$ is the NS shear viscosity. The latter quantity is given, in the simplest Sonine approximation, by [2]

$$
\eta_{\mathrm{NS}}=\frac{n T}{v+\zeta / 2}, \quad v=\frac{1+\alpha}{2}\left[1-\frac{d-1}{2 d}(1-\alpha)\right] \omega .
$$

The uniform longitudinal viscous flow is an unsteady compressible flow defined by a linear longitudinal velocity field, a uniform density, and a uniform temperature [3]. The exact balance equations for mass and energy yield

$$
u_{x}(x, t)=a(t) x, \quad n(t)=\frac{n_{0}}{a_{0}} a(t), \quad a(t)=\frac{a_{0}}{1+a_{0} t}
$$



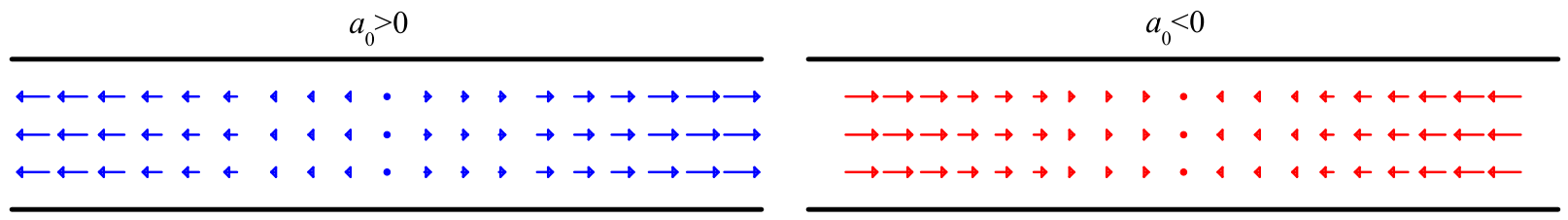

FIGURE 1. Sketch of the uniform longitudinal flow for $a_{0}>0$ (left) and $a_{0}<0$ (right).

$$
\partial_{t} T(t)=-\frac{2 a_{0}}{d n_{0}} P_{x x}(t)-\zeta(t) T(t)
$$

This simple flow is also known as homo-energetic extension and, along with the uniform shear flow [4, 5], is a particular case of a more general class of homo-energetic affine flows characterized by $\partial^{2} u_{i} / \partial x_{j} \partial x_{k}=0$ [4]. The initial longitudinal deformation rate rate $a_{0}$ is the only control parameter determining the departure of the fluid from the homogeneous state, thus playing a role similar to that of the shear rate in the uniform shear flow state. On the other hand, in contrast to the uniform shear flow, the sign of $a_{0}$ plays a relevant role and defines two distinct situations (see Fig. 1). The case $a_{0}>0$ corresponds to a progressively slower expansion of the gas from the plane $x=0$ into all of space. On the other hand, the case $a_{0}<0$ corresponds to a progressively faster compression of the gas towards the plane $x=0$. The latter takes place over a finite time period $t=\left|a_{0}\right|^{-1}$. However, since the collision frequency rapidly increases with time, the finite period $t=\left|a_{0}\right|^{-1}$ comprises an infinite number of collisions per particle (see below).

According to Newton's law (2), the normal stress $P_{x x}$ in the uniform longitudinal flow is given by

$$
T_{x}^{*}(t) \equiv \frac{P_{x x}(t)}{p(t)}=1-2 \frac{d-1}{d} \frac{a^{*}(t)}{1+\zeta^{*} / 2}, \quad a^{*}(t) \equiv \frac{a(t)}{v(t)} \propto \frac{a_{0}}{n_{0}} \frac{1}{\sqrt{T(t)}},
$$

where $\zeta^{*} \equiv \zeta / v$ is a dimensionless constant (except that it depends on $d$ and $\alpha$ ). Note that $T_{x}^{*}$ is the ratio between the "longitudinal" temperature $T_{x}=P_{x x} / n$ and the true granular temperature $T$. The absolute value of the reduced longitudinal rate $a^{*}$ plays the role of the Knudsen number of the problem. The linear relationship (6) between $T_{x}^{*}$ and $a^{*}$ is expected to hold for small values of $\left|a^{*}\right|$ only. More in general, one can define a (reduced) nonlinear viscosity function $\eta^{*}\left(a^{*}\right)$ by the relation

$$
T_{x}^{*}(t)=1-2 \frac{d-1}{d} \eta^{*}\left(a^{*}(t)\right) a^{*}(t), \quad \lim _{a^{*} \rightarrow 0} \eta^{*}\left(a^{*}\right)=\frac{1}{1+\zeta^{*} / 2} \equiv c_{0} .
$$

The expansion of $\eta^{*}\left(a^{*}\right)$ in powers of $a^{*}$ yields the CE series

$$
\eta^{*}\left(a^{*}\right)=\sum_{k=0}^{\infty} c_{k} a^{* k}
$$

where $c_{1}$ is a Burnett coefficient, $c_{2}$ is a super-Burnett coefficient, and so on.

In this paper a kinetic theory description of the longitudinal viscous flow defined by Eqs. (4) and (5) is presented. As will be shown, a steady-state value of the temperature is reached only if $a_{0}<0$. The main result is the derivation of a nonlinear first-order differential equation for $\eta^{*}\left(a^{*}\right)$. This equation allows one to get the CE coefficients $c_{k}$ of Eq. 8) in a recursive way. The results show that the $\mathrm{CE}$ series is convergent for inelastic hard spheres, while it diverges in the elastic case. The viscosity function $\eta^{*}\left(a^{*}\right)$ for any value of the inelasticity parameter $\alpha$ can be obtained by numerically solving the differential equation with appropriate boundary conditions. Finally, a simplified analytical solution hardly distinguishable from the numerical solution is proposed.

\section{KINETIC MODEL}

In order to obtain explicit results, it is convenient to consider the following BGK-like kinetic model [6] of the inelastic Boltzmann equation:

$$
\left(\partial_{t}+\mathbf{v} \cdot \nabla\right) f=-v\left(f-f_{\mathrm{hcs}}\right)+\frac{\zeta}{2} \partial_{\mathbf{v}} \cdot[(\mathbf{v}-\mathbf{u}) f]
$$


where $f$ is the velocity distribution function and $f_{\text {hcs }}$ is the local version of the homogeneous cooling state distribution [1]. The NS viscosity coincides with that of the Boltzmann equation if $v$ is given by Eq. (3). However, a simpler and more consistent choice is $v=\frac{1}{2}(1+\alpha) \omega$ [7], so that $\zeta^{*}=[(d+2) / 2 d](1-\alpha)$. In the uniform longitudinal flow the velocity distribution function $f(x, \mathbf{v}, t)$ becomes spatially uniform when the velocities are referred to a Lagrangian frame moving with the flow, i.e., $f(x, \mathbf{v}, t)=f(\mathbf{V}, t)$, where $\mathbf{V} \equiv \mathbf{v}-\mathbf{u}(x, t)$ is the peculiar velocity. After simple algebra, Eq. (9) can be rewritten as

$$
\left(\partial_{\tau}-a_{0} \partial_{V_{x}} V_{x}\right) \bar{f}=-\bar{v}\left(\bar{f}-\bar{f}_{\mathrm{hcs}}\right)+\frac{\bar{\zeta}}{2} \partial_{\mathbf{v}} \cdot(\mathbf{V} \bar{f})
$$

where

$$
\bar{f}(\mathbf{V}, \tau) \equiv \frac{n_{0}}{n(t)} f(x, \mathbf{v}, t), \quad \bar{v}(\tau) \equiv \frac{n_{0}}{n(t)} v(t), \quad \bar{\zeta}(\tau) \equiv \frac{n_{0}}{n(t)} \zeta(t), \quad \tau \equiv a_{0}^{-1} \ln \left(1+a_{0} t\right) .
$$

Equation (10) shows that the original uniform longitudinal flow problem can be mapped onto the equivalent problem of a uniform gas with a velocity distribution $\bar{f}$ and subject to the action of a non-conservative force $-m a_{0} V_{x} \widehat{\mathbf{x}}$. The density associated with $\bar{f}$ is the initial density $n_{0}$ and thus remains constant. In fact, $\bar{f}$ is the probability distribution function normalized to $n_{0}$. The time variable $\tau=\int_{0}^{t} \mathrm{~d} t^{\prime} n\left(t^{\prime}\right) / n_{0}$ is a nonlinear measure of time scaled with the number density. It is unbounded even if $a_{0}<0$ since in that case $\tau \rightarrow \infty$ when $t \rightarrow\left|a_{0}\right|^{-1}$.

Taking velocity moments on both sides of Eq. (10) one gets

$$
\partial_{\tau} T+\frac{2}{d} a_{0} T_{x}=-\bar{\zeta} T, \quad \partial_{\tau} T_{x}+2 a_{0} T_{x}=-\bar{v}\left(T_{x}-T\right)-\bar{\zeta} T_{x} .
$$

If $a_{0}>0, \partial_{\tau} T<0$ even in the elastic case $(\zeta=0)$. As a consequence, the gas cools down and the reduced longitudinal rate $a^{*}=a / v \sim 1 / \sqrt{T}$ increases without bound, i.e., $\lim _{\tau \rightarrow \infty} a^{*}(\tau)=\infty$. On the other hand, in the compression case $\left(a_{0}<0\right)$ the "viscous heating" term $(2 / d)\left|a_{0}\right| T_{x}$ competes with the "inelastic cooling" term $\bar{\zeta} T$, so that the temperature either decreases or increases with time depending on whether in the initial state one has $\bar{\zeta} T>(2 / d)\left|a_{0}\right| T_{x}$ or $\bar{\zeta} T<(2 / d)\left|a_{0}\right| T_{x}$, respectively. In either case, a steady-state temperature $T_{s}$ is eventually reached when both effects cancel each other. Therefore, if $a_{0}<0$ one has $\lim _{\tau \rightarrow \infty} a^{*}(\tau)=a_{s}^{*}$. The steady-state values for the reduced longitudinal rate $a^{*}$, the temperature ratio $T_{x} / T$, and the reduced viscosity $\eta^{*}$ can be easily obtained from Eq. (12) and are given by

$$
a_{s}^{*}=-\frac{d}{2} \zeta^{*} \frac{1+\zeta^{*}}{1+d \zeta^{*}}, \quad T_{x, s}^{*}=\frac{1+d \zeta^{*}}{1+\zeta^{*}}, \quad \eta_{s}^{*}=\frac{1+d \zeta^{*}}{\left(1+\zeta^{*}\right)^{2}} .
$$

Comparison between $\eta_{s}^{*}$ and the NS value $c_{0}$ in Eq. (7) shows that the steady state is inherently non-Newtonian, even in the quasi-elastic limit $\zeta^{*} \ll 1$, in which case $c_{0} \approx 1-\zeta^{*} / 2$ whilst $\eta_{s}^{*} \approx 1+(d-2) \zeta^{*}$. Note that in the elastic case $(\zeta=0)$ with $a_{0}<0$ only the viscous heating term is present in the energy balance equation, so that $\lim _{\tau \rightarrow \infty} a^{*}(\tau)=0$.

In summary, the zero Knudsen number value $\left(a^{*}=0\right)$ is a "repeller" of the time evolution of $a^{*}(\tau)$ in the inelastic case. For elastic collisions, the state $a^{*}=0$ is also a repeller if $a_{0}>$ but it is an "attractor" of $a^{*}(\tau)$ if $a_{0}<0$. Figure 2 a) shows $a_{s}^{*}, T_{x, s}^{*}$, and $\eta_{s}^{*}$ as functions of $\alpha$ for three-dimensional systems $(d=3)$. It is interesting to note that the steady-state reduced viscosity $\eta_{s}^{*}$ is nearly independent of $\alpha$.

As noted before, if $a_{0}<0$ the whole compression process takes place during a finite time period $t=\left|a_{0}\right|^{-1}$. Let us see now that the corresponding number of collisions per particle is infinite. The number of collisions per particle during an elementary time interval $\mathrm{d} t$ is $\mathrm{d} s=v \mathrm{~d} t=\bar{v} \mathrm{~d} \tau$. The accumulated number of collisions between the initial state and the scaled time $\tau$ is then $s(\tau)=v_{0} \int_{0}^{\tau} \mathrm{d} \tau^{\prime} \sqrt{T\left(\tau^{\prime}\right) / T_{0}}$, where $v_{0}$ and $T_{0}$ are the initial collision frequency and temperature, respectively. If $a_{0}<0$ the temperature is bounded between $T_{0}$ and $T_{s}$, i.e., $\min \left(T_{0}, T_{s}\right) \leq T\left(\tau^{\prime}\right) \leq \max \left(T_{0}, T_{s}\right)$. Therefore, $\min \left(1, \sqrt{T_{s} / T_{0}}\right) v_{0} \tau \leq s(\tau) \leq \max \left(1, \sqrt{T_{s} / T_{0}}\right) v_{0} \tau$. Since $\tau \rightarrow \infty$ when $t \rightarrow\left|a_{0}\right|^{-1}$, it follows that $s \rightarrow \infty$ in that limit.

\section{NONLINEAR VISCOSITY}

In order to get the whole rheological function $\eta^{*}\left(a^{*}\right)$ we need to eliminate time in favor of $a^{*}(\tau)=a_{0} / \bar{v}(\tau)$, taking into account that both $\bar{v}$ and $\bar{\zeta}$ are proportional to $[T(\tau)]^{q}$, where $q=\frac{1}{2}$. It is convenient to consider for the moment $q$ as an arbitrary positive parameter and set $q=\frac{1}{2}$ at the end. Thus, since $T_{x}=T_{x}^{*} T$, one has

$$
\partial_{\tau} T_{x}=T_{x}^{*} \partial_{\tau} T+T\left(\partial_{\tau} a^{*}\right) \partial_{a^{*}} T_{x}^{*}=\left(T_{x}^{*}-q a^{*} \partial_{a^{*}} T_{x}^{*}\right) \partial_{\tau} T .
$$



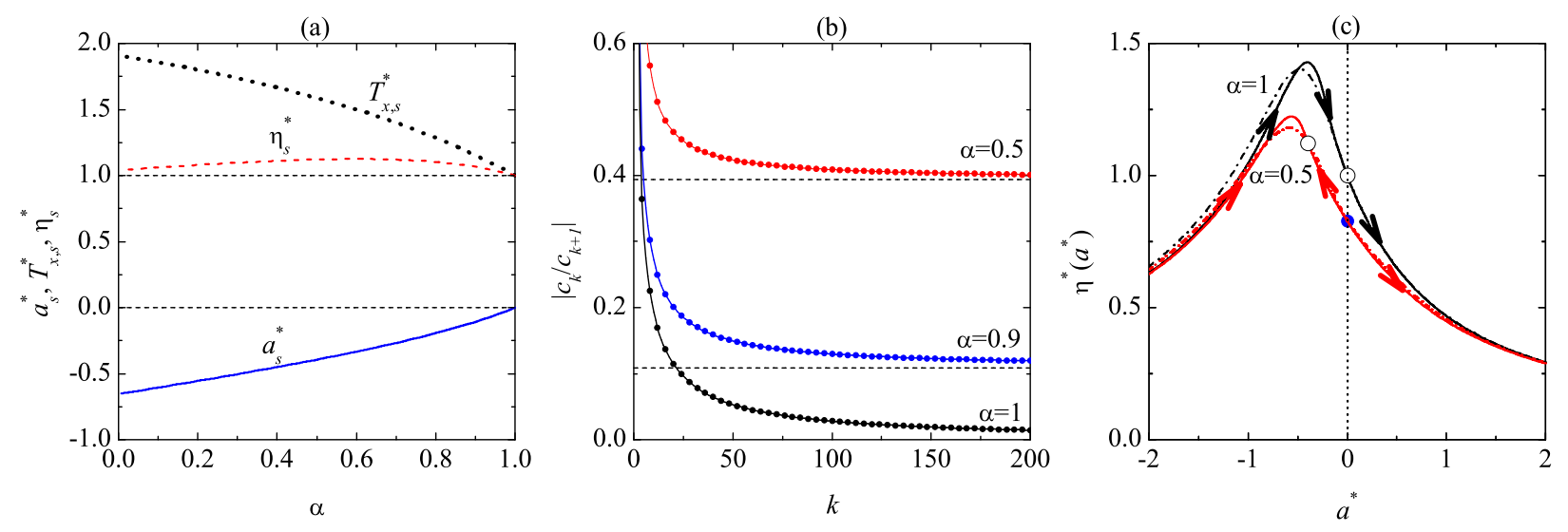

FIGURE 2. (a) Plot of the steady-sate values $a_{s}^{*}, T_{x, s}^{*}$, and $\eta_{s}^{*}$ as functions of $\alpha$. (b) Ratio $\left|c_{k} / c_{k+1}\right|$ for $\alpha=0.5,0.9$, and 1; the horizontal dashed lines indicate the corresponding values of $\left|a_{s}^{*}\right|$. (c) Nonlinear viscosity for $\alpha=0.5$ and 1 as obtained from the numerical solution of Eq. [15] (solid lines) and from Eq. (20) (dash-dotted lines); the circles denote the steady-state points and the arrows indicate the direction of the time evolution. All the panels correspond to three-dimensional hard spheres $\left(q=\frac{1}{2}, d=3\right)$.

Making use of Eq. (12) it is easy to get the following nonlinear first-order differential equation for the function $\eta^{*}\left(a^{*}\right)$ :

$$
\eta^{*}-\left(1-2 \frac{d-1}{d} \eta^{*} a^{*}\right)\left(1+\frac{2}{d} \eta^{*} a^{*}\right)+q\left[\zeta^{*}+\frac{2}{d} a^{*}\left(1-2 \frac{d-1}{d} \eta^{*} a^{*}\right)\right]\left(\eta^{*}+a^{*} \partial_{a^{*}} \eta^{*}\right)=0 .
$$

Inserting the $\mathrm{CE}$ expansion $(8)$ one can get the coefficients $c_{k}$ in a recursive way:

$$
c_{0}=\frac{1}{1+q \zeta^{*}}, \quad c_{k}=-\frac{2}{d} \frac{1}{1+(k+1) q \zeta^{*}}\left\{(d-2+k q) c_{k-1}+2 \frac{d-1}{d} \sum_{\ell=0}^{k-2} c_{\ell} c_{k-2-\ell}[1-(\ell+1) q]\right\} .
$$

Figure 2 (b) shows the absolute value of the ratio $c_{k} / c_{k+1}$ for three-dimensional hard spheres $\left(q=\frac{1}{2}, d=3\right)$ with $\alpha=0.5$ (highly inelastic case), $\alpha=0.9$ (moderately inelastic case), and $\alpha=1$ (elastic case). In the inelastic cases the ratio $\left|c_{k} / c_{k+1}\right|$ tends to a non-zero finite value, thus indicating that the CE series (8) converges. In fact, the results are consistent with $\lim _{k \rightarrow \infty}\left|c_{k} / c_{k+1}\right|=\left|a_{s}^{*}\right|$, i.e., the radius of convergence of the CE series coincides with the steadystate value of the Knudsen number. In contrast, in the elastic case one has $\lim _{k \rightarrow \infty}\left|c_{k} / c_{k+1}\right|=0$, so that the radius of convergence shrinks to zero and the CE series (16) diverges. In fact, if $\zeta^{*}=0$, the magnitude of the coefficients $c_{k}$ grows so fast that the summation term in Eq. (15) can be neglected and one has $\left|c_{k} / c_{k+1}\right| \approx(d / 2 q) k^{-1}$.

Inspection of Eq. (15) shows the following asymptotic behaviors in the limit of $\left|a^{*}\right| \rightarrow \infty$ :

$$
\begin{array}{ll}
a_{0}>0: & \lim _{a^{*} \rightarrow \infty} \eta^{*}\left(a^{*}\right) a^{*}=\frac{d}{2(d-1)}, \quad \lim _{a^{*} \rightarrow \infty} T_{x}^{*}\left(a^{*}\right)=0, \\
a_{0}<0: & \lim _{a^{*} \rightarrow-\infty} \eta^{*}\left(a^{*}\right) a^{*}=-\frac{d}{2}, \quad \lim _{a^{*} \rightarrow-\infty} T_{x}^{*}\left(a^{*}\right)=d .
\end{array}
$$

Therefore, in the limit $a^{*} \rightarrow \infty$ there is no motion (relative to the flow velocity) along the $x$ direction, while in the opposite limit $a^{*} \rightarrow-\infty$ the motion becomes one-dimensional along the $x$ axis.

The complete viscosity function $\eta^{*}\left(a^{*}\right)$ for any $a^{*}$, including values of the Knudsen number $\left|a^{*}\right|$ beyond the radius of convergence $\left|a_{s}^{*}\right|$, is obtained by numerically solving the differential equation (15) with appropriate boundary conditions. To get the branches $a^{*}>0$ and $a_{s}^{*}<a^{*}<0$ one must start from a small value of $\left|a^{*}\right|$ and apply the initial condition $\eta^{*}=c_{0}+c_{1} a^{*}$. The branch $a^{*}<a_{s}^{*}<0$ is obtained by starting from a large value of $a^{*}$ and applying the boundary condition $\eta^{*}=-d / 2 a^{*}$. The resulting curve $\eta^{*}\left(a^{*}\right)$ is plotted in Fig. 2 (2) for three-dimensional hard spheres $\left(q=\frac{1}{2}, d=3\right)$ with $\alpha=0.5$ and 1 . The arrows on the curves indicate the direction followed by the time evolution of $a^{*}(\tau)$.

While the differential equation (15) can be solved numerically, it is interesting to approximate its solution by an analytical expression. To that end, let us see $q$ as a small perturbation parameter and formally expand the solution to Eq. (15) in powers of $q$ :

$$
\eta^{*}\left(a^{*}\right)=\eta_{0}^{*}\left(a^{*}\right)\left[1-q h_{1}\left(a^{*}\right)+\cdots\right] .
$$




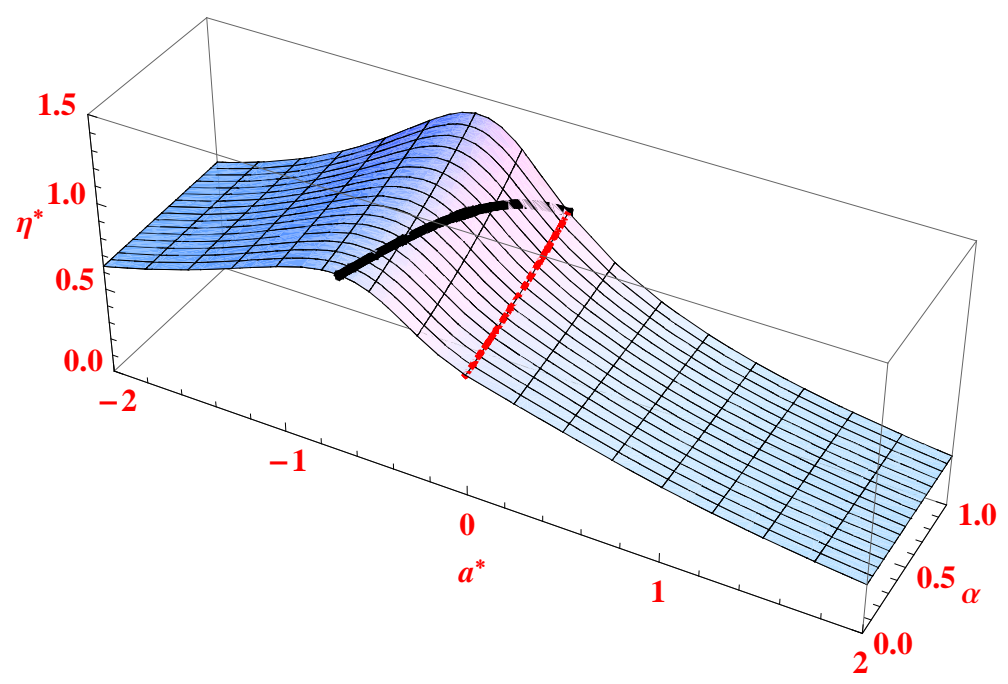

FIGURE 3. Surface plot showing the dependence on both $a^{*}$ and $\alpha$ of of the viscosity function $\eta^{*}$ given by Eq. (20). The thin and thick lines refer to the NS and steady-state points, respectively.

By inserting Eq. (18) into Eq. (15) and keeping terms linear in $q$, one gets, after some algebra,

$$
\eta_{0}^{*}\left(a^{*}\right)=\left[\frac{1}{2}+\frac{d-2}{d} a^{*}+\beta\left(a^{*}\right)\right]^{-1}, \quad h_{1}\left(a^{*}\right)=\frac{\zeta^{*}+\frac{1}{2}+a^{*}-\beta\left(a^{*}\right)}{4 \beta^{2}\left(a^{*}\right)}, \quad \beta\left(a^{*}\right) \equiv \sqrt{\left(\frac{1}{2}+a^{*}\right)^{2}-\frac{2}{d} a^{*}} .
$$

Note that the zeroth-order function $\eta_{0}^{*}\left(a^{*}\right)$ is independent of the reduced cooling rate $\zeta^{*}$. Instead of proceeding to higher order terms in the $q$-expansion (18), let us consider the simple Padé approximant

$$
\eta^{*}\left(a^{*}\right) \simeq \frac{\eta_{0}^{*}\left(a^{*}\right)}{1+q h_{1}\left(a^{*}\right)} .
$$

This approximation has the merit of providing correctly (for any value of $q$ ) the values of the NS coefficient $c_{0}$ [see Eq. (16)] and the steady-state point $\eta_{s}^{*}=\eta^{*}\left(a_{s}^{*}\right)$ [see Eq. (13)], as well as the limiting behaviors (17). Moreover, even in the physical case of hard spheres $\left(q=\frac{1}{2}\right)$, Eq. (20) represents an excellent analytical approximation to the numerical solution of Eq. (15), especially for highly inelastic systems, as shown in Fig. 2(c). The discrepancies are only apparent near the maximum of $\eta^{*}\left(a^{*}\right)$ and in the decay to the left of the maximum. Figure 3 shows a surface plot of Eq. (20).

\section{CONCLUSIONS}

The uniform longitudinal viscous flow is an unsteady compressible flow [see Eqs. (4) and (5)] that, despite its apparent simplicity, constitutes a non-trivial playground for nonequilibrium statistical mechanics beyond the NS description. While this state has been studied in the past for normal gases [3], the present work addresses the problem in the case of a granular gas of inelastic hard spheres. The relevant control parameter is the reduced longitudinal rate $a^{*}(t)=a(t) / v(t)$ (whose magnitude plays the role of the Knudsen number of the problem) and the relevant response function is the timedependent generalized viscosity $\eta^{*}\left(a^{*}\right)$ defined by Eq. (7). The exact energy balance equation (5) shows that if $a_{0}>0$ (expansion states), the temperature monotonically decreases and so $a^{*}(t)$ increases with time, for both elastic $(\zeta=0)$ and inelastic $(\zeta>0)$ collisions. On the other hand, if $a_{0}<0$ (compression states), $a^{*}(t)$ goes to zero from below in the elastic case, whereas it tends to a stationary value $a_{s}^{*}<0$ in the inelastic case [see Fig. 2(a)]. Expressed in other terms, one can say that the homogeneous state $\left(a_{0}=0\right.$ or $\left.a^{*}=0\right)$ is unstable against any perturbation $a_{0} \neq 0$, no matter how weak it is, if the particles are inelastic. On the other hand, $a^{*}=0$ is stable for elastic collisions if $a_{0}<0$. Given that the CE expansion (8) is carried out about (and measures the departure from) the reference homogeneous state $\left(a^{*}=0\right)$, it follows the "arrow of time" for granular gases but goes against it for normal gases (if $a_{0}<0$ ). As a consequence, it can be expected on physical grounds that the CE expansion diverges in the case of elastic collisions but does converge if the collisions are inelastic, the radius of convergence $\left|a_{s}^{*}\right|$ increasing with inelasticity. 
To confirm the above expectation and also to get the rheological function $\eta^{*}\left(a^{*}\right)$, the simple model kinetic equation (9) has been considered in this paper. The viscosity function $\eta^{*}\left(a^{*}\right)$ predicted by the model is the solution to the nonlinear first-order differential equation (15) (with $q=\frac{1}{2}$ ). The CE coefficients $c_{k}$ are shown to verify the asymptotic law $\lim _{k \rightarrow \infty}\left|c_{k} / c_{k+1}\right|=\left|a_{s}^{*}\right|$, thus confirming the convergence of the series (8) with a finite radius $a^{*}=\left|a_{s}^{*}\right|$, which vanishes in the elastic limit [see Fig.2(b)]. The function $\eta^{*}\left(a^{*}\right)$ exhibits an interesting behavior [see Fig.2(c)]. Starting from the NS value $\eta^{*}(0)=c_{0}$, it decreases as the longitudinal rate $a^{*}$ increases, behaving as $\eta^{*}\left(a^{*}\right) \approx[d / 2(d-1)] / a^{*}$ in the limit of large positive $a^{*}$. In the latter limit, the gas becomes highly anisotropic with no random motion along the flow direction. The situation is even more interesting in the case of negative $a^{*}$. As the magnitude of the longitudinal rate increases, the viscosity function starts increasing, reaches a maximum value past the steady-state point, and decreases thereafter, behaving as $\eta^{*}\left(a^{*}\right) \approx(d / 2) /\left|a^{*}\right|$ in the limit of large $\left|a^{*}\right|$. The gas becomes highly anisotropic also in this limit, but this time because the degrees of freedom orthogonal to the flow direction are suppressed. As Fig. 2 (c) shows, the largest quantitative influence of inelasticity on the shape of $\eta^{*}\left(a^{*}\right)$ occurs near the maximum. It is also in this region where the analytical approximation (20) exhibits the largest deviations from the numerical solution of Eq. (15). Otherwise, Eq. (20) succeeds in capturing the main features of the solution, including the steady-state and NS values, as well as the asymptotic behaviors. However, an important property not accounted for by Eq. (15) is the divergence of the CE series in the elastic case $\left(\zeta^{*}=0\right)$. This is a consequence of the fact that Eq. (15) is based on the $q$-expansion (18) (truncated after the first term) and the CE expansion in the reference system $(q=0)$ is convergent, regardless the value of $\zeta^{*}$.

As a final point, it is worthwhile rewriting the CE expansion $(8)$ in the dimensional form

$$
P_{x x}=p-2 \frac{d-1}{d} \eta_{\mathrm{NS}} \sum_{k=0}^{\infty} \frac{c_{k}}{v^{k} c_{0}}\left(\frac{\partial u_{x}}{\partial x}\right)^{k+1} .
$$

When arbitrary hydrodynamic gradients are present, Eq. (21) represents a partial contribution to the general CE expansion of the normal stress $P_{x x}$. The advantage of the uniform longitudinal flow is that it possesses an only hydrodynamic gradient, $\partial u_{x} / \partial x$, which is spatially uniform. Therefore, in this case the general CE expansion of $P_{x x}$ reduces to Eq. (21). However, the convergence or divergence of the partial series (21) does not depend on whether the system is actually in the uniform longitudinal flow or in any other state. According to the results derived in this paper, it turns out that the partial series (21) diverges for normal gases but converges for granular gases. A similar conclusion is reached in the case of the expansion of the shear stress $P_{x y}$ in powers of the shear rate $\partial u_{x} / \partial y[8]$.

\section{ACKNOWLEDGMENTS}

This work has been supported by the Ministerio de Educación y Ciencia (Spain) through Grant No. FIS2007-60977 (partially financed by FEDER funds) and by the Junta de Extremadura (Spain) through Grant No. GRU08069.

\section{REFERENCES}

1. N. Brilliantov and T. Pöschel, Kinetic Theory of Granular Gases, Oxford U. P., Oxford, 2004; C. S. Campbell, Annu. Rev. Fluid Mech. 22, 57-92 (1990); J. W. Dufty, J. Phys.: Condens. Matt. 12 A47-A56 (2000); I. Goldhirsch, Annu. Rev. Fluid Mech. 35, 267-293 (2003).

2. J. J. Brey, J. W. Dufty, C. S. Kim, and A. Santos, Phys. Rev. E 58, 4638-4653 (1998).

3. A. N. Gorban and I. V. Karlin, Phys. Rev. Lett. 77, 282-285 (1996); I. V. Karlin, G. Dukek, and T. F. Nonnenmacher, Phys. Rev. E 55, 1573-1576 (1997); F. J. Uribe and E. Piña, Phys. Rev. E 57, 3672-3673 (1998); I. V. Karlin, G. Dukek, and T. F. Nonnenmacher, Phys. Rev. E 57, 3674-3675 (1998); F. J. Uribe and L. S. García-Colín, Phys. Rev. E 60, 4052-4062 (1999); A. Santos, Phys. Rev. E 62, 6597-6607 (2000).

4. C. Truesdell and R. G. Muncaster, Fundamentals of Maxwell's Kinetic Theory of a Simple Monatomic Gas, Academic Press, New York, 1980.

5. V. Garzó and A. Santos, Kinetic Theory of Gases in Shear Flows. Nonlinear Transport Kluwer Academic Publishers, Dordrecht, 2003.

6. J. J. Brey, J. W. Dufty, and A. Santos, J. Stat. Phys. 97, 281-322 (1999).

7. A. Santos and A. Astillero, Phys. Rev. E 72, 031308-1-19 (2005); F. Vega Reyes, V. Garzó, and A. Santos, Phys. Rev. E 75, 061306-1-14 (2007).

8. A. Santos, Phys. Rev. Lett. 100, 078003-1-4 (2008); A. Santos, "Does the Chapman-Enskog expansion for viscous granular flows converge?," to appear in Proceedings of the XVth International Congress on Rheology, AIP Conference Proceedings; preprint arXiv:0804.2566 